\title{
Percutaneous Coronary Intervention for a Patient with Left Main Coronary Compression Syndrome
}

\author{
Ryutaro Ikegami, Kazuyuki Ozaki, Takuya Ozawa, Satoru Hirono, \\ Masahiro Ito and Tohru Minamino
}

\begin{abstract}
:
Left main coronary compression syndrome rarely occurs in patients with severe pulmonary hypertension. A 65-year-old woman with severe pulmonary hypertension due to an atrial septal defect suffered from angina on effort. Cardiac computed-tomography and coronary angiography revealed considerable stenosis of the left main coronary artery (LMA) caused by compression between the dilated main pulmonary artery trunk and the sinus of valsalva. Stenting of the LMA under intravascular ultrasound imaging was effective for the treatment of angina. We herein report the diagnosis and management of this condition with a brief literature review.
\end{abstract}

Key words: pulmonary hypertension, Eisenmenger syndrome, dilated main pulmonary artery, revascularization for left main coronary artery

(Intern Med 57: 1421-1424, 2018)

(DOI: 10.2169/internalmedicine.9534-17)

\section{Introduction}

Patients with longstanding and severe pulmonary hypertension $(\mathrm{PH})$ often have a markedly dilated pulmonary artery. The enlargement of the main pulmonary artery trunk rarely but occasionally causes compression of the left main coronary artery (LMA) in these patients (1-5). This finding is described as 'left main coronary artery compression syndrome (LMCS)' and is known to lead to angina, acute myocardial infarction, worsening heart failure, and sudden cardiac death. In addition, congenital heart disease with a left to right shunt flow is known to cause $\mathrm{PH}$, and without appropriate treatment, eventually results in Eisenmeger syndrome. In 1989, Mitsudo et al. reported a series of angiography findings showing LMA narrowing due to compression from the dilated pulmonary artery in patients with an atrial septal defect (ASD) (2). In general, the first choice of management for an unprotected LMA lesion is coronary artery bypass grafting $(\mathrm{CABG})(6)$.

However, there are some reports of percutaneous coronary intervention (PCI) with LMCS patients due to their high surgical mortality (7-13). Similarly, our present experience showed that PCI with stenting was effective for protecting against lumen collapse and resolving angina.

\section{Case Report}

A 65-year-old woman suffered from chest pain on effort, and her condition was complicated by congestive heart failure (CHF). She had been diagnosed with an ASD in childhood. Several years earlier, she had visited our hospital with a complaint of shortness of breath and was found to already have severe pulmonary hypertension $(\mathrm{PH})$ with decreased oxygen saturation on effort. After an examination, including cardiac catheterization, she was diagnosed with Eisenmenger syndrome due to the ASD and judged not to be a surgical candidate for closure of the ASD. Therefore, she was provided with home oxygen therapy and $\mathrm{PH}$-specific medications.

After stabilization of her CHF, coronary computedtomography angiography (CTA) was performed because she had symptom of chest compression on effort, such as when she walked fast or did strenuous housework. CTA revealed narrowing of the LMA due to compression between the enlarged pulmonary artery trunk and the valsalva sinus 

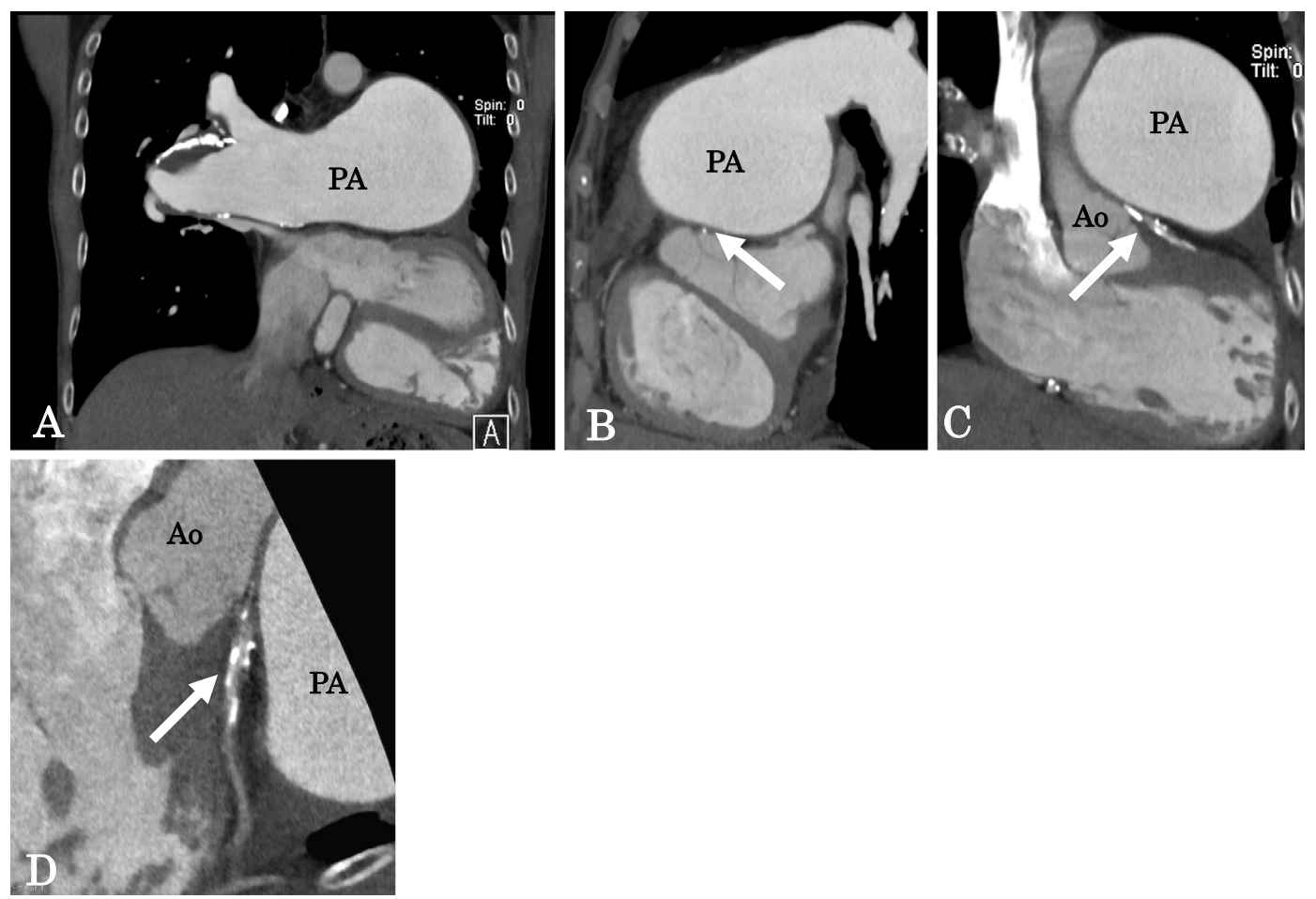

Figure 1. (A) CT showed marked enlargement of the pulmonary artery due to severe PH. (B, C, D) The compression of the LMCA (white arrow) by the dilated pulmonary artery trunk and sinus of valsalva was confirmed by CTA.
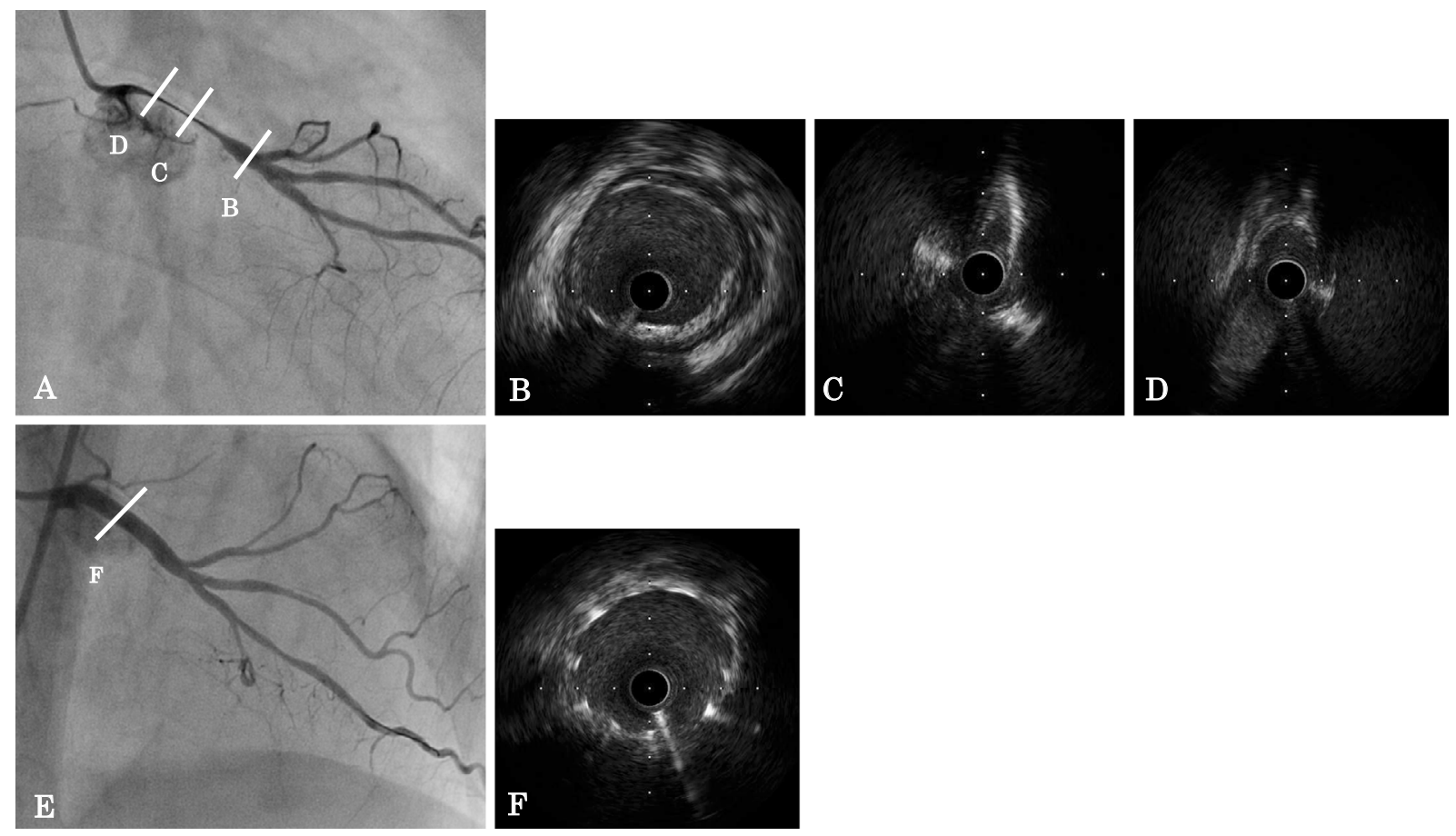

Figure 2. (A) Cardiac angiography confirmed narrowing of the LMCA by $90 \%$. (B, C, D) IVUS demonstrated no significant atheroma in the LAD proximal portion. (B) From the proximal LAD to the LMCA ostium, there was superficial calcification and deformation with a slit-like appearance due to compression by the reduced echoic space in both sides. (E, F) Post-stenting angiography and IVUS showed the complete resolution of the LMCA stenosis.

(Fig. 1). Cardiac angiography (CAG) showed that her right coronary was dominant with hypoplasia of the left circumflex artery and that she had severe stenosis extending from the ostium of the LMA to the proximal portion of the left anterior descending artery (LAD) (Fig. 2A). The pressure data were as follows: arterial pressure 93/59 (70) $\mathrm{mmHg}$, 


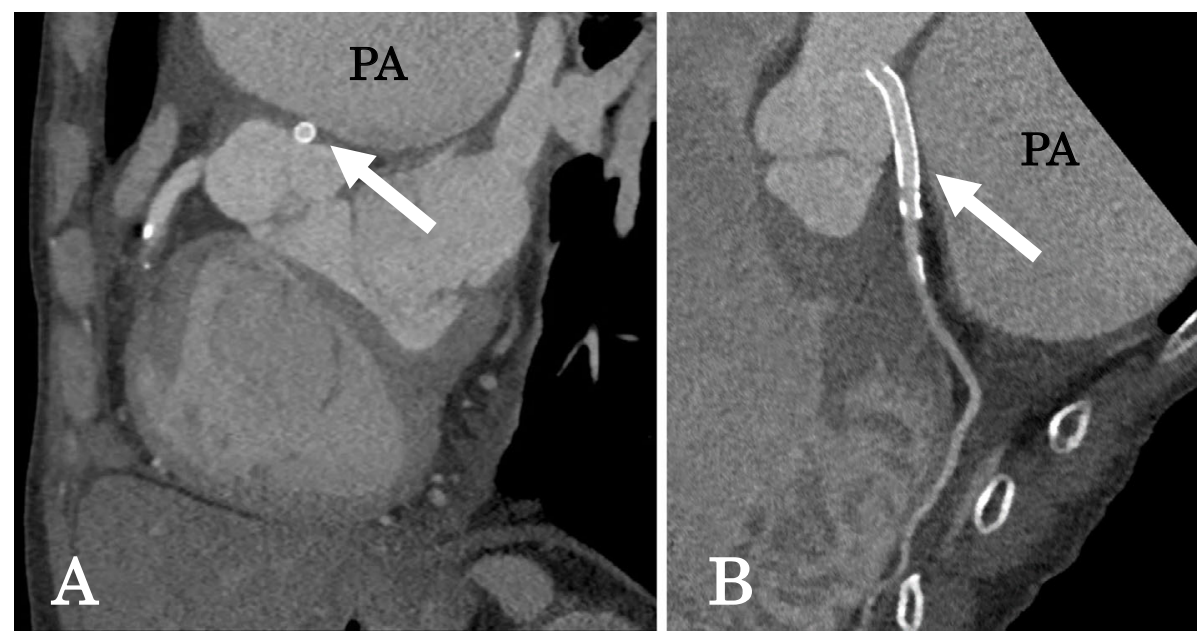

Figure 3. (A, B) The stent patency at three months after the PCI was confirmed by coronary CTA (white arrow).

pulmonary artery pressure $66 / 34(45) \mathrm{mmHg}$, and cardiac output index $1.61 \mathrm{~L} / \mathrm{min} / \mathrm{m}^{2}$. She had no coronary risk. Therefore, we diagnosed her with effort-induced angina due to left main compression syndrome. We concluded that the revascularization of the LMA was necessary and that PCI with stenting was recommended over CABG because surgery under general anesthesia was considered too risky.

PCI for the LMA lesion due to extrinsic compression was performed. A 7-Fr JL3.5 guiding catheter was inserted via the right femoral artery. After crossing the guide wire to the LAD, intravascular ultrasound imaging (IVUS) was performed, showing no stenosis from the distal to the proximal portion of the LAD and a lumen diameter of $4.0 \mathrm{~mm}$ (Fig. 2B-D). From the ostium of the LMA to the proximal LAD, LMA deformation was apparent with a slit-like appearance due to compression by a reduced echoic space, indicating fluid on both sides. IVUS revealed the following: minimum lumen diameter, shortest $1.16 \mathrm{~mm}$ and longest $2.24 \mathrm{~mm}$; and minimum lumen area, $1.56 \mathrm{~mm}^{2}$. There were no arteriosclerotic plaques and only mild superficial calcification at the lesion. A bare-metal stent (Integrity 4.0×26 $\mathrm{mm}$; Medtronic, Minneapolis, USA) was implanted. Final CAG showed favorable dilation of the LMA lesion (Fig. 2E), and IVUS showed good expansion of the stent with optimal placement (Fig. 2F). The PCI procedure was completed without any complications. After PCI, the patient no longer experienced angina. She was already being administered anti-coagulation therapy with warfarin for pulmonary hypertension, and dual anti-platelet therapy was started additionally. One month after PCI, anti-platelet therapy was changed to a single anti-platelet regimen with aspirin. We confirmed the stent patency by CTA after three months (Fig. 3). Follow-up angiography was performed at six months and showed neither compression nor restenosis.

\section{Discussion}

LMA compression due to an enlarged pulmonary artery was first described by Corday et al. in 1957 (1). Since then, there have been several reports of LMCS as a complication of PH (2-5, 7-18). The major etiology of LMCS is secondary $\mathrm{PH}$ due to congenital heart disease, including ASD, patent ductus arteriosus, ventricular septum defect, and Tetralogy of Fallot (5). Recently, an LMCS patient with chronic thromboembolic pulmonary hypertension was described (12). Vaseghi et al. found that PH patients with small stature were predisposed to coronary compression (13). In their case series, the median height of their patients was $160 \mathrm{~cm}$ (range: 154-167 cm). Coronary compression is likely associated with the diameter of mediastinum. Our patient, whose body height was $156 \mathrm{~cm}$, was also of small stature.

PH patients often have symptoms of angina without coronary stenosis because of right ventricular ischemia due to high pulmonary arterial pressure (14). However, several reports described LMCS due to severe PH. Mesquita et al. reported that 19 out of 36 patients with $\mathrm{PH}$ without stenosis and with coronary artery compression had symptoms of angina (15). Coronary CTA is a useful non-invasive way of detecting LMCS with $\mathrm{PH}$, as it can reveal both the enlargement of the pulmonary artery and the compression of the coronary artery. This report suggested that LMA compression was more likely to occur in the patients with a diameter of their pulmonary trunk $>4.0 \mathrm{~cm}$ and a pulmonary trunk-toaortic diameter ratio $>1.2$ (15). Another report found that an angle of the LMA with the left sinus of valsalva $<30^{\circ}$ increased the likelihood of significant myocardial ischemia (8). In our case, the diameter of the pulmonary trunk was $6.3 \mathrm{~cm}$, the pulmonary trunk-to-aortic diameter ratio was 2.8 , and the angle was $24^{\circ}$, all of which placed our patient in the high-risk category for LMCS. Coronary CTA is therefore helpful for the diagnosis of LMCS.

If the extrinsic compression of the LMA causes myocardial ischemia, coronary revascularization should be considered necessary. There was a report of such a case progressing to acute coronary syndrome (16). The standard method 
of revascularization for unprotected LMA disease is CABG (6). Several reports have indicated the efficacy of CABG for patients with LMCS $(17,18)$. However, surgical treatment is not indicated in severe $\mathrm{PH}$ patients because of the high risk of surgical mortality. In 2001, Rich et al. reported for the first time excellent results for PCI with stenting for patients with LMCS (7). Since then, there have been several reports of successful PCI cases in similar patients (7-13). In these reports, either bare-metal stents or drug-eluting stents were used, and the stent type did not influence the results. Although stent durability under this condition is unknown, there are no reports of problems, including stent fracture. The longest follow up after stent implantation was three years. Stent selection should be made based on the vessel diameter, arteriosclerotic changes in the coronary artery, and risk of bleeding complications. In our case, the IVUS findings showed that the vessel size was over 4.0 $\mathrm{mm}$ and that there was little plaque in the coronary artery. In addition, patients with Eisenmenger syndrome and $\mathrm{PH}$ patients taking either anti-coagulation or prostaglandin medication are at risk of hemoptysis (19). Therefore, we selected a bare-metal stent that was shorter in duration (only for one month) and administered dual anti-platelet therapy instead of using a drug-eluting stent.

In summary, we described a case of angina due to LMCS and performed PCI with stenting successfully. Non-invasive coronary CTA was useful for detecting the dilation of the pulmonary artery and compression of the coronary artery. Stent implantation is a feasible option for the treatment of LMCS, specifically for patients with a high surgical risk.

For studies with human subjects, include the following statement before the References section: All procedures followed were in accordance with the ethical standards of the responsible committee on human experimentation (institutional and national) and with the Helsinki Declaration of 1964 and later revision. Informed consent or substitute for it was obtained from all patients for being included in the study.

The authors state that they have no Conflict of Interest (COI).

\section{References}

1. Corday E, Gold H, Kaplan L. Coronary artery compression; an explanation for the cause of coronary insufficiency in pulmonary hypertension. Trans Am Coll Cardiol 7: 93-103, 1957.

2. Mitsudo K, Fujino T, Matsunaga K, et al. Coronary arteriographic findings in the patients with atrial septal defect and pulmonary hypertension $(\mathrm{ASD}+\mathrm{PH})$-compression of left main coronary artery by pulmonary trunk. Kokyu To Junkan 37: 649-655, 1989 (in Japanese, Abstract in English).

3. Fujiwara K, Naito Y, Higashiue S. Left main coronary trunk compression by dilated main pulmonary artery in atrial septal defect. Report of three cases. J Thorac Cardiovasc Surg 104: 449-452, 1992.

4. Kawut SM, Silvestry FE, Ferrari VA, et al. Extrinsic compression of the left main coronary artery by the pulmonary artery in patients with long-standing pulmonary hypertension. Am J Cardiol 83: 984-986, 1999.

5. Kajita LJ, Martnez EE, Ambrose JA, et al. Extrinsic compression of the left main coronary artery by a dilated pulmonary artery: clinical, angiographic, and hemodynamic determinants. Catheter Cardiovase Interv 52: 49-54, 2001.

6. Fihn SD, Gardin JM, Abrams J, et al. ACCF/AHA/ACP/AATS/ PCNA/SCAI/STS guideline for the diagnosis and management of patients with stable ischemic heart disease: executive summary: a report of the American College of Cardiology Foundation/American Heart Association task force on practice guidelines, and the American College of Physicians, American Association for Thoracic Surgery, Preventive Cardiovascular Nurses Association, Society for Cardiovascular Angiography and Interventions, and Society of Thoracic Surgeons. Circulation 126: 3097-3137, 2012.

7. Rich S, McLaughlin VV, O'Neill W. Stenting to reverse left ventricular ischemia due to left main coronary artery compression in primary pulmonary hypertension. Chest 120: 1412-1415, 2001.

8. Dodd JD, Maree A, Paracios I, et al. Left main coronary artery compression syndrome: evaluation with 64-slice cardiac multidetector computed tomography. Circulation 115: e7-e8, 2007.

9. Sivakumar K, Rajan M, Francis G, Murali K, Bashi V. Extrinsic compression of the left coronary ostium by the pulmonary trunk: management in a case of Eisenmenger syndrome. Tex Heart Inst J 37: 95-98, 2010.

10. Caldera AE, Cruz-Gonzalez I, Bezerra HG, Cury RC, Palacios IF, Cockrill BA. Endovascular therapy for left main compression syndrome. Case report and literature review. Chest 135: 1648-1650, 2009.

11. Andjelkovic K, Kalimanovaska-Ostric D, Djyukic M, et al. Two rare conditions in an Eisenmenger patient: left main coronary artery compression and Ortner's syndrome due to pulmonary artery dilatation. Heart Lung 42: 382-386, 2013.

12. Bonderman D, Fleischmann D, Prokop M, Klepetko W, Lang IM. Left main coronary artery compression by the pulmonary trunk in pulmonary hypertension. Circulation 105: 265, 2002.

13. Vaseghi M, Lee SM, Currier J, Tobis J, Shapiro S, Aboulhosn J. Percutaneous intervention of left main coronary artery compression by pulmonary artery aneurysm. Catheter Cardiovasc Interv 76: 352-356, 2010.

14. Rich S. Primary pulmonary hypertension. Prog Cardiovasc Dis 31: 205-238, 1988.

15. Mesquita SM, Castro CR, Ikari NM, Oliveira SA, Lopes AA. Likelihood of left main coronary artery compression based on pulmonary trunk diameter in patients with pulmonary hypertension. Am J Med 116: 369-374, 2004.

16. Lindsey JB, Brilakis ES, Banerjee S. Acute coronary syndrome due to extrinsic compression of the left main coronary artery in a patient with severe pulmonary hypertension: successful treatment with percutaneous coronary intervention. Cardiovasc Rev Med 9: 47-51, 2008.

17. Lee J, Kwon HM, Hong BK. Total occlusion of left main coronary artery by dilated main pulmonary artery in a patient with severe pulmonary hypertension. Korean J Intern M 16: 265-269, 2001.

18. Kito K, Fujiwara Y, Kimura T, Shimada Y. Left main coronary trunk compression by dilated main pulmonary artery in a patient with atrial septal defect. Masui 50: 184-187, 2001 (in Japanese, Abstract in English).

19. Daliento L, Somerville J, Presbitero P, et al. Eisenmenger syndrome. Factors relating to deterioration and death. Eur Heart J 19: 1845-1855, 1998.

The Internal Medicine is an Open Access article distributed under the Creative Commons Attribution-NonCommercial-NoDerivatives 4.0 International License. To view the details of this license, please visit (https://creativecommons.org/licenses/ by-nc-nd/4.0/).

(C) 2018 The Japanese Society of Internal Medicine

Intern Med 57: 1421-1424, 2018 